\title{
Preparation of Nickel Chloride of High Purity
}

\author{
W. Stanley Clabaugh, John W. Donovan, and Raleigh Gilchrist
}

\begin{abstract}
A method is described for the preparation of cobalt-free nickel chloride, which consists in the repeated precipitation by hydrogen chloride of nickel chloride dihydrate from a mixture of a saturated aqueous solution of nickel chloride with acetone. Methods are also given for determining cobalt, aluminum, iron, copper, and combined phosphorus and silicon in nickel salts.
\end{abstract}

\section{Introduction}

In developing methods of testing nickel salts used as analytical reagent-grade chemicals, it became necessary to prepare nickel compounds that were free of cobalt. Most reagent-grade nickel salts contain approximately 0.2 percent of cobalt, although a special low-cobalt nickel sulfate, containing 0.002 to 0.005 percent of cobalt, is available.

The literature revealed no reliable process for preparing nickel salts free of cobalt. However, three papers did have a bearing on the subject. Garwin and Hixson, ${ }^{1}$ in a study of the separation of cobalt from nickel by extraction from aqueous solution, found that anhydrous cobalt chloride was soluble in organic solvents, such as certain aliphatic alcohols, ketones, esters, and acids. They state that the addition of an electrolyte, hydrogen chloride in particular, greatly increases the efficiency of the extractive separation.

Tillu ${ }^{2}$ developed an analytical separation of cobalt from nickel, based on the solubility of anhydrous cobalt chloride in acetone.

Katzin and Ferraro ${ }^{3}$ studied the systems, cobaltous nitrate-water-acetone and cobaltous nitrate-water-tbutyl alcohol, with respect to types of compounds formed to gain information to account for the high solubility of inorganic salts in certain types of organic solvents.

The following procedure embraces the general principles involved in the above-mentioned papers. In addition to eliminating cobalt, the procedure also reduces or eliminates other impurities, such as aluminum, copper, iron, etc.

\section{Procedure for Removing Cobalt from Nickel Chloride}

Prepare a nearly saturated solution of nickel chloride in water at room temperature. Add a volume of acetone equal to approximately four times the volume of the aqueous nickel solution. The two liquids are only very slightly miscible under these conditions.

Warm the mixture on the steam bath to a temperature just below the boiling point of the acetone. Pass a rapid stream of hydrogen-chloride gas into the lower aqueous layer until the nickel chloride

\footnotetext{
L. Garwin and A. Hixson, Ind. Eng. Chem. 41, 2298, 2303“(1949).

${ }^{2}$ M. M. Tillu, J. Indian Chem. Soc. 20, 139 (1943)

${ }^{3}$ I. Katzin and J. R. Ferraro, J. Am. Chem. Soc. 72, 5451 (1950).
}

precipitates as a dihydrate and the two liquid phases become one. Decant most of the supernatant liquid, collect the nickel salt on a suction filter, and wash it with acetone.

If the operation is performed at a temperature just below the boiling point of the acetone, the nickel salt separates in a fine crystalline form that is very easy to filter and to wash. The product formed at room temperature appears to consist of a mixture of hydrates that separate in large aggregates of moist crystals. This type of precipitate has a tendency to cohere into one solid mass at the bottom of the container. Precipitation at the higher temperature increases the vield of nickel salt, furnishes a product that is easy to handle, but does not significantly affect the efficiency of the separation.

\section{Purification of Nickel Chloride}

One kilogram of nickel chloride hexahydrate, which contained 0.17 percent of cobalt, was subjected to four successive precipitations in the manner described above. The over-all yield of high-purity nickel salt was approximately 85 percent. Because of the great solubility of nickel chloride, the volumes of the solutions were relatively small, so that the precipitating operation could be performed in a 4-liter beaker.

The original material taken, when calculated as the dihydrate, contained 2,400 parts of cobalt per million. The first precipitation reduced the cobalt content to $130 \mathrm{ppm}$; the second, to $6.4 \mathrm{ppm}$; the third, to about 0.2 or $0.3 \mathrm{ppm}$. Cobalt was not detected in the fourth precipitate either chemically, by the method described later, or spectrochemically.

In addition to removing the cobalt, it was found that the aluminum content dropped from 4 to $1 \mathrm{ppm}$; copper, from 16 to $0.4 \mathrm{ppm}$; and iron, from 1 to less than $0.1 \mathrm{ppm}$. These values, determined chemically, were confirmed by independent spectrochemical analysis. Spectrochemical test showed that calcium and magnesium each was present in the final product to the extent of from 1 to $10 \mathrm{ppm}$. The combined phosphorus and silicon content in the final product was found chemically to be less than $1 \mathrm{ppm}$, calculated as silicon.

The nickel, chlorine, and water contents of the purified product were found to be $35.4,42.4$, and 21.0 percent, respectively, as compared with the calculated values of $35.4,42.8$, and 21.7 percent for $\mathrm{NiCl}_{2} \cdot 2 \mathrm{H}_{2} \mathrm{O}$. 


\section{Methods of Determining Impurities}

\subsection{Determination of Cobalt}

Cobalt was determined by means of a potentiometric titration in which bivalent cobalt is oxidized to tervalent cobalt by potassium ferricyanide. The method used was an adaptation of that developed by Tomicek and Freiberger. ${ }^{4}$

Any manganese present will be titrated and reported as cobalt. ${ }^{5}$

Tomicek was able to estimate as little as $15 \mu \mathrm{g}$ of cobalt in the presence of $0.2 \mathrm{~g}$ of nickel. ${ }^{6}$ The present authors were able to determine as little as 2 $\mu \mathrm{g}$ of cobalt in the presence of $2 \mathrm{~g}$ of nickel. The accuracy of the determination for amounts of cobalt less than $20 \mu \mathrm{g}$ appeared to be $\pm 0.5 \mu \mathrm{g}$.

In adapting the method of Tomicek to this work, the present authors found that the potential at the end point of the titration depends on the concentration of ammonia and ammonium chloride. Large amounts of ammonia lower the potential, whereas ammonium chloride increases it. Ammonium chloride tends to stabilize the potential, especially during stirring of the solution. If the proper concentrations of ammonia and ammonium chloride are established, the end point of the titration may be taken as that point at which the potential of the solution is zero when compared to the potential of a saturated calomel electrode. Although this method of determining the end point may introduce a small error, usually less than $10 \mu \mathrm{g}$ of cobalt, it does serve as a rapid routine method for determining fairly small amounts. For the accurate determination of very small amounts of cobalt, the values of the potentials must be plotted and the exact end point determined graphically. In the present work, measurements of the voltage were made with a high-impedance input vacuum-tube voltmeter.

Because the presence of even small amounts of oxygen interferes with the titration, all the solutions must be prepared with oxygen-free water, and protected during the titration. A suitable titration assembly consists of a $100-\mathrm{ml}$ beaker with a closefitting rubber stopper, through which are holes for inserting the calomel and platinum electrodes, buret tip, and a tube for passing in inert gas. The amount of ferricyanide added can be easily controlled if the buret is connected by means of a short piece of transparent tubing to a glass tube that is drawn to a fine tip. It is this latter tip that should be inserted through the rubber stopper. The above arrangement will allow the solution to be stirred by swirling the contents of the beaker.

In determining microgram quantities of cobalt, a 0.001- $M$ solution of ferricyanide is used, whereas for larger quantities a $0.02-M$ concentration is satisfac-

${ }^{4} \mathrm{O}$. Tomicek and F. Freiberger, J. Am. Chem. Soc. 57, 801 (1935).

5 O. Tomicek and J. Kalny, J. Am. Chem. Soc. 57, 1209 (1935).

6 O. Tomicek, Chem. Listy 36, 44 (1942). tory. The nickel salt may be either the chloride, nitrate, sulfate, or acetate, etc., provided sufficient ammonia is added to convert all the nickel to the cation $\left[\mathrm{Ni}\left(\mathrm{NH}_{3}\right)_{6}\right]^{++}$and to provide a final concentration of ammonia of about $4 \mathrm{~N}$.

In the determination of cobalt in the nickel chloride dihydrate, $2 \mathrm{~g}$ of the salt was dissolved in $10 \mathrm{ml}$ of water in the $100-\mathrm{ml}$ titration beaker. After inserting the rubber stopper containing the electrodes, etc., carbon dioxide was passed through the solution for 10 to $20 \mathrm{~min}$, after which $20 \mathrm{ml}$ of an ammoniaammonium chloride solution, made by dissolving 6 g of $\mathrm{NH}_{4} \mathrm{Cl}$ in $100 \mathrm{ml}$ of $6 \mathrm{~N} \mathrm{NH}$ $\mathrm{NH}_{3}$ ), was added. The flow of carbon dioxide was stopped and the cobalt titrated with ferricyanide.

To ascertain whether known quantities of cobalt, when added to the cobalt-free nickel chloride, could be accounted for, $2.0,3.0,5.0$, and $10.0 \mu \mathrm{g}$ of cobalt each added to $2 \mathrm{~g}$ of the dihydrate gave 1.7, 2.8, 5.0, and $10.0 \mu \mathrm{g}$, respectively.

\subsection{Determination of Other Impurities}

Aluminum was determined according to the general method of Gentry and Sherrington, ${ }^{7}$ which consists in complexing the nickel with cyanide in ammoniacal solution, and extracting the aluminum with a 1-percent solution of 8-hydroxyquinoline in chloroform. The amount of aluminum was ascertained by measuring the absorbancy of the chloroform extract at $395 \mathrm{~m} \mu$. The value so obtained was corrected for a small amount of iron, which was also extracted, by measuring the absorbancy of the same extract at $580 \mathrm{~m} \mu .^{8}$

Iron was determined by measuring the absorbancy of the isoamyl alcohol extract of the thiocyanate complex.

Copper was determined by extracting with a 0.002 -percent solution of dithizone in carbon tetrachloride, from a solution containing $0.05 \mathrm{~N}$ acid, to eliminate any interference by nickel, and by measuring the absorbancy of the extract.

The combined phosphorus and silicon content was determined by measuring the absorbancy of the "molybdenum blue" that was produced. The heteropolycomplex with molybdenum was formed in ammoniacal solution. The solution was then acidified and filtered to remove any nickel ammonium sulfate. The combined heteropolycomplexes were extracted with isoamyl alcohol and reduced to molybdenum blue by stannous chloride. The color produced was compared with those of known amounts of silicon, as silicate, treated in the same manner as the sample.

7 C. H. R. Gentry and L. G. Sherrington, The Analyst r1, 432 (†946). 8 T. Moeller, Ind. Eng. Chem., Anal. Ed. 15, 346 (1943).

Washington, July 16, 1953. 